\title{
Vascular Vertigo
}

Mazyar Hashemilar ${ }^{1}$, M asoud Nikanfar ${ }^{1}$, Dariush Savadi Oskoui ${ }^{1}$

${ }^{1}$ Department of Neurology, Tabriz University of M edical Sciences, Tabriz, Iran.

Vertigo is a common complaint in neurology and medicine. The most common causes of vertigo are benign paroxysmal positional vertigo, vestibular neuritis, Meniere's disease, and vascular disorders. Vertigo of vascular origin is usually limited to migraine, transient ischemic attacks, and ischemic or hemorrhagic stroke. Vascular causes lead to various central or peripheral vestibular syndromes with vertigo. This review provides an overview of epidemiology and clinical syndromes of vascular vertigo.

Vertigo is an illusion of movement caused by asymmetrical involvement of the vestibular system by various causes. M igraine is the most frequent vascular disorder that causes vertigo in all age groups. Vertigo may occur in up to $25 \%$ of patients with migraine. The lifetime prevalence of migrainous vertigo is almost $1 \%$. Cerebrovascular disorders are estimated to account for $3 \%$ to $7 \%$ of patients with vertigo. Vestibular paroxysmia has been diagnosed in $1.8 \%$ to $4 \%$ of cases in various dizziness units. Vasculitic disorders are rare in the general population, but vertigo may be seen in almost up to $50 \%$ of patients with different vasculitic syndromes.

Conclusions: Migraine, cerebrovascular disorders especially involving the vertebrobasilar territory, cardiocirculatory diseases, neurovascular compression of the eighth nerve, and vasculitis are vascular causes of vertigo syndromes.

Key words: vascular vertigo, epidemiology, migrainous vertigo, vertebrobasilar insufficiency, vestibular paroxysmia, vasculitis

DOI: $10.7575 /$ aiac.abcmed.ca1.21

Published Date: February 2017

Peer-review is under responsibility of the 9th Iranian Stroke Congress.

Published by Australian International Academic Centre, Australia

This published work is open access under the CC BY license.

Available online at www.abcmed.aiac.org.au 